\title{
Rapid Detection and Differentiation of Swine-Origin Influenza A Virus (H1N1/2009) from Other Seasonal Influenza A Viruses
}

\author{
Jiangqin Zhao ${ }^{1}{ }^{*}$, Xue Wang ${ }^{1}$, Viswanath Ragupathy ${ }^{1}$, Panhe Zhang ${ }^{1}$, Wei Tang ${ }^{1}$, \\ Zhiping Ye ${ }^{2}$, Maryna Eichelberger ${ }^{2}$ and Indira Hewlett ${ }^{1, *}$
}

1 Laboratory of Molecular Virology, Center for Biologics Evaluation and Research, Food and Drug Administration, Bethesda, MD 20892, USA; E-Mails: xue.wang@fda.hhs.gov (X.W.); viswanath.ragupathy@fda.hhs.gov (V.R.); panhe.zhang@fda.hhs.gov (P.Z.); annietang_93@yahoo.com (W.T.)

2 Division of Viral Products, Office of Vaccines Research and Review, CBER, Food and Drug Administration, Bethesda, MD 20892, USA; E-Mails: zhiping.ye@fda.hhs.gov (Z.Y.); maryna.eichelberger@fda.hhs.gov (M.E.)

* Authors to whom correspondence should be addressed; E-Mails: jiangqin.zhao@fda.hhs.gov (J.Z.); indira.hewlett@fda.hhs.gov (I.H.); Tel.: +1-301-827-0795; Fax: +1-301-480-7928.

Received: 25 August 2012; in revised form: 1 November 2012 / Accepted: 1 November 2012 / Published: 9 November 2012

\begin{abstract}
We previously developed a rapid and simple gold nanoparticle(NP)-based genomic microarray assay for identification of the avian $\mathrm{H} 5 \mathrm{~N} 1$ virus and its discrimination from other influenza A virus strains (H1N1, H3N2). In this study, we expanded the platform to detect the 2009 swine-origin influenza A virus (H1N1/2009). Multiple specific capture and intermediate oligonucleotides were designed for the matrix $(\mathrm{M})$, hemagglutinin (HA), and neuraminidase (NA) genes of the H1N1/2009 virus. The H1N1/2009 microarrays were printed in the same format as those of the seasonal influenza H1N1 and H3N2 for the HA, NA, and M genes. Viral RNA was tested using capture-target-intermediate oligonucleotide hybridization and gold NP-mediated silver staining. The signal from the 4 capture-target-intermediates of the HA and NA genes was specific for H1N1/2009 virus and showed no cross hybridization with viral RNA from other influenza strains H1N1, H3N2, and H5N1. All of the $3 \mathrm{M}$ gene captures showed strong affinity with H1N1/2009 viral RNA, with 2 out of the $3 \mathrm{M}$ gene captures showing cross hybridization with the H1N1, H3N2, and H5N1 samples tested. The current assay was able to detect H1N1/2009 and distinguish it from other influenza A viruses. This new method may be useful for simultaneous detection and subtyping of influenza A viruses and can be rapidly modified
\end{abstract}


to detect other emerging influenza strains in public health settings.

Keywords: nanoparticle; H5N1; swine influenza A virus; nanomicroarray

\section{Introduction}

Influenza viruses are classified as $\mathrm{A}, \mathrm{B}$, and $\mathrm{C}$ based on antigenic differences in their nucleoprotein (NP) and matrix (M) protein. Influenza A viruses are classified into 16 HA subtypes (H1-H16) and 9 NA subtypes (N1-N9) based on the antigenicity of the two surface glycoproteins: hemagglutinin (HA) and neuraminidase (NA). All subtypes of influenza A virus are found in aquatic birds, while only a subset cause disease in pigs, horses, dogs, sea mammals, and humans [1-3]. HA subtypes (H1, H2, and H3) and two NA subtypes (N1 and N2) have caused human pandemics [4,5]. Influenza viruses of $\mathrm{H} 5$ and $\mathrm{H} 7$ subtypes are highly pathogenic to domestic fowl due to the presence of a multibasic tryptic cleavage site in the HA [6,7]. Although it is not easily transmissible between people, avian H5N1 viruses have infected humans with significant rate of mortality since 1997 [8]. In addition to direct infection of man by avian viruses, novel genotypic reassortants in an intermediary host may result in the emergence of a novel pandemic strain. In mid-March 2009, a novel swine-origin influenza virus entered the human population and spread rapidly around the world. WHO received global data for large numbers of cases and deaths due to this 2009 swine-origin influenza virus (H1N1/2009) early on in the pandemic [9], and the virus has continued to circulate in humans, replacing the previously circulating seasonal H1N1 viruses. Continued reassortment of H1N1/2009 with swine influenza viruses may produce variants with transmissibility and altered virulence for humans [10,11]. To identify emerging viruses with pandemic potential, molecular virological monitoring needs to be implemented as a means to assess the extent to which novel strains are circulating in a population.

Rapid subtyping of influenza A viruses, such as the pandemic H1N1/2009 and the avian influenza H5N1 viruses, is crucial to initiate adequate protective measures to prevent the spread of highly pathogenic influenza A viruses. At the present time, the most commonly used diagnostic and subtyping methods are time consuming, expensive, and inaccurate. By using a nanoparticle(NP)-based PCR-free genomic microarray assay (nanomicroarray), we previously developed a H5N1 microarray to rapidly and specifically detect H5N1 viral RNA [12]. In this study we describe the development of the nanomicroarray assay for rapid detection and subtyping of the H1N1/2009 influenza virus that discriminates between this and other seasonal influenza A viruses.

\section{Results and Discussion}

To verify the ability to detect $\mathrm{H} 1 \mathrm{N1} / 2009$ viral RNA, the nanomicroarray was initially designed and evaluated using PCR amplicons for the $\mathrm{H} 1 \mathrm{~N} 1 / 2009 \mathrm{H} 1, \mathrm{~N} 1$, and $\mathrm{M}$ genes $\left(\mathrm{H} 1_{09}, \mathrm{~N}_{09}, \mathrm{M}_{09}\right)$. The assay was performed by direct hybridization of PCR products with the capture and the intermediate oligonucleotides to form sandwich complexes, followed by silver staining in a format shown in Figure 1A, with each H1N1/2009 capture (swc) oligonucleotide, listed in Table 1, spotted in triplicate. As shown in Figure 1B, PCR-amplified $\mathrm{H} 1_{09}, \mathrm{~N} 1_{09}$, and $\mathrm{M}_{09}$ genes resulted in signals that 
corresponded with the capture oligonucleotide, showing specificity of the assay, and providing a unique fingerprint for each gene segment. The inability of capture swcH1 to detect the $\mathrm{H} 1_{09}$ PCR amplicon is likely reflective of incorrect oligonucleotide synthesis. Analytical specificity was demonstrated by mixing the three DNA products simultaneously during hybridization. The signal patterns observed for the $\mathrm{H}_{09}, \mathrm{~N}_{109}$, and $\mathrm{M}_{09}$ genes when used either in separate assays or together, were the same, although in the mixture, the $\mathrm{M}_{09}$ gene had low intensity. When the PCR products of the HA and NA genes for the seasonal H1N1, H3N2, and H5N1 subtypes were tested in a H1N1/2009 array, no signal was detected (data not shown). This study indicates that the H1N1/2009 array is highly specific for the detection of HA and NA genes from only the swine-origin influenza A virus.

Figure 1. Image from a Verigene $\mathrm{ID}^{\mathrm{TM}}$ detection system. (A) Nanomicroarray layout with positive control capture (closed red circles), negative control (closed blank circles), M, HA, and NA gene captures (filled as variable color of closed circles, spotted in triplicate) are indicated. The numbers indicate the different capture sequences from a gene shown in Table 1. Three types of arrays for H1N1, H3N2, and H1N1/2009 are printed in the same format as indicated. (B) Silver staining image for individual or multiple H1N1/2009 PCR products are shown and labeled. (C) Silver staining image from $0.5 \mu \mathrm{g}$ of viral RNA for H1N1, H3N2, H5N1, and H1N1/2009 are shown.
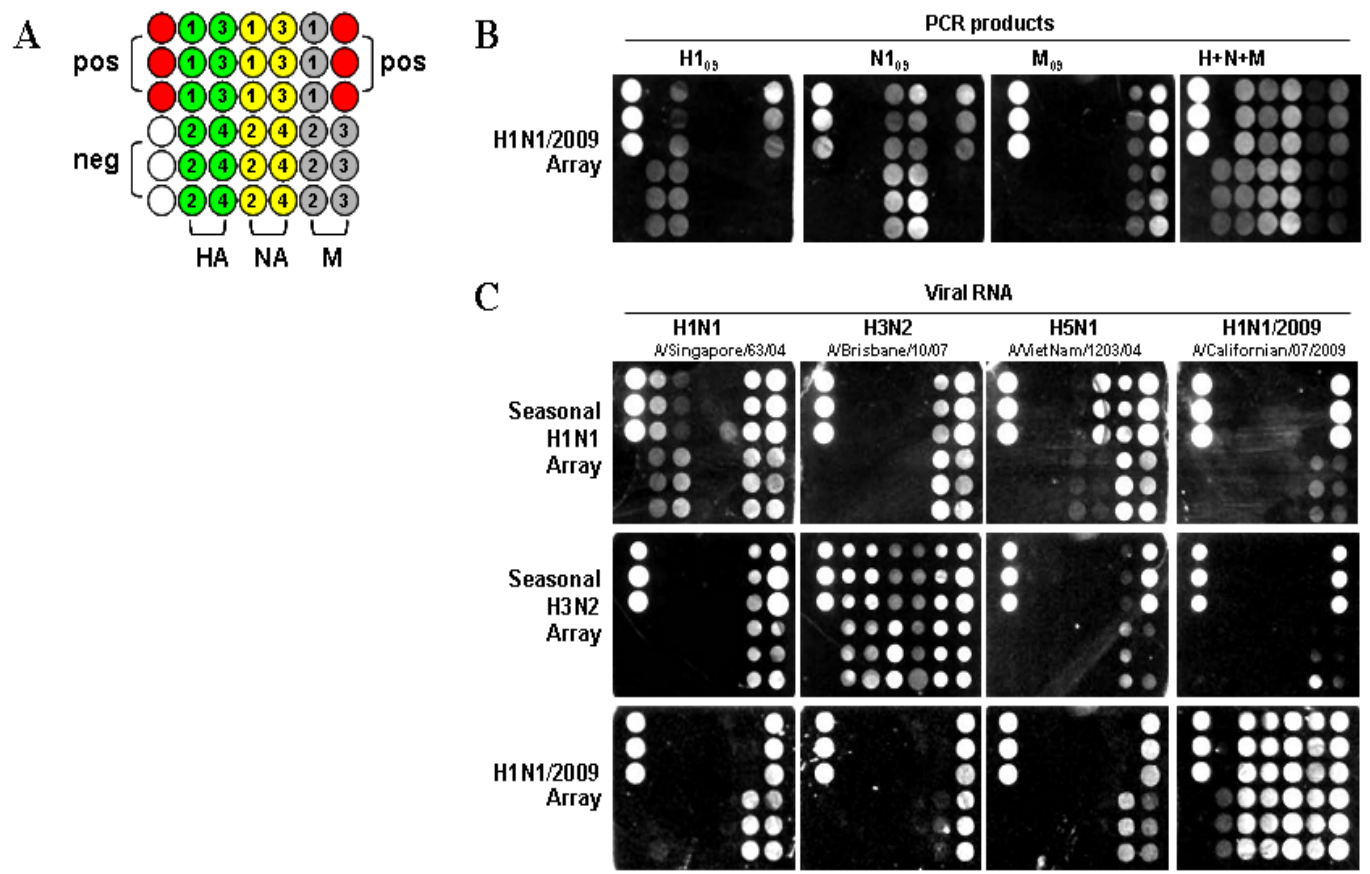

To determine the ability to differentiate seasonal H1N1 and H3N2 from the H1N1/2009 subtype, three types of nanomicroarray were generated. RNA samples extracted from seasonal H1N1, H3N2, avian H5N1, and H1N1/2009 viruses listed in the Experimental Section were tested in those arrays (Figure 1C). In the seasonal H1N1 array, only the HA gene from A/Singapore/63/04 (H1N1) was detected but not from the $\mathrm{H} 1 \mathrm{~N} 1 / 2009$ strain, A/Californian/07/2009. The NA gene from A/VietNam/1203/04 (H5N1) and A/Singapore/63/04 (H1N1) were detected by this array, but there was 
no cross-reactivity with the H1N1/2009 NA gene. In the seasonal H3N2 array, all three genes (H3, N2, and $\mathrm{M}$ ) were detected in the $\mathrm{A} /$ Brisbane/10/07 (H3N2) sample.

In the H1N1/2009 array, viral RNA extracted from three H1N1/2009 strains (A/California/04/2009, $\mathrm{A} /$ California/07/2009 and A/California/08/2009) were tested and a similar signal pattern was observed. As shown in Figure $1 \mathrm{C}$, all three genes $\left(\mathrm{H}_{09}, \mathrm{N1}_{09}\right.$, and $\left.\mathrm{M}_{09}\right)$ from the A/California/07/2009 (H1N1) sample displayed strong signals. The three $\mathrm{H}_{09}$ capture oligonucleotides ( $\mathrm{swcH} 2, \mathrm{swcH} 3$ and swcH4) and four $\mathrm{N}_{109}$ capture oligonucleotides ( $\mathrm{swcN} 1, \operatorname{swcN} 2, \operatorname{swcN} 3$ and $\left.s w c N 4\right)$ could efficiently detect H1N1/2009 strain. There was only one capture swcH1 that did not produce any signal as described above in PCR amplicons suggesting that errors may have occurred in this capture oligonucleotide, a new synthesized capture swcH1 needs further evaluation. The array format described here was specifically designed to evaluate individual design of capture oligonucleotides, synthesis and printing for detection of H1N1/2009. The array has since been printed multiple times and was shown to be highly reproducible between independent arrays. This assay has a feature for detection of a specific target gene (i.e., $\mathrm{H}_{09}$ ) through multiple capture oligonucleotides and does not rely on any single capture such as swcH1. The functional capture oligonucleotides evaluated in this study were used in the development of a multiplex nanomicroarray for detection and differentiation of influenza A from B and subtyping influenza A viruses.

Table 1. Oligonucleotide sequences for H1N1/2009 capture (swc), intermediate (swi) and PCR.

\begin{tabular}{|c|c|c|c|}
\hline Oligo ID & Gene & Purpose & Sequences (5' to 3') \\
\hline SwcM1 & $\mathrm{M}$ & capture & CTGACTAAGGGAATTTTAGGATTTGTGTTCACGCTCACCG \\
\hline swcM2 & M & capture & TGATTCACAGCATCGGTCTCACAGACAGATGGCTACTACC \\
\hline swcM3 & M & capture & AAGGCTATGGAACAGATGGCTGGATCGAGTGAACAGGCAG \\
\hline swcH1 & HA & capture & GAAGGCAATACTAGTAGTTCTGCTATATACATTTGCAACC \\
\hline $\mathrm{swcH} 2$ & HA & capture & CCTGGGAAATCCAGAGTGTGAATCACTCTCCACAGCAAGC \\
\hline swcH3 & HA & capture & GTTTTTGTGGGGTCATCAAGATACAGCAAGAAGTTCAAGC \\
\hline swcH4 & HA & capture & GAAGCAAAATTAAACAGAGAAGAAATAGATGGGGTAAAGC \\
\hline swcN1 & NA & capture & TTCGGTCTGTATGACAATTGGAATGGCTAACTTAATATTA \\
\hline swcN2 & NA & capture & GTGGTTTCCGTGAAATTAGCGGGCAATTCCTCTCTCTGCC \\
\hline swcN3 & NA & capture & CAATGGAACCATTAAAGACAGGAGCCCATATCGAACCCTA \\
\hline swcN4 & NA & capture & ACGCCCTAATGATAAGACAGGCAGTTGTGGTCCAGTATCG \\
\hline swiM1 & M & intermediate & AAAGCCGAGATCGCGCAGAGACTGGAAAGTGTCTTTGCAG * \\
\hline swiM2 & M & intermediate & CCACTAATCAGGCATGAAAACAGAATGGTGCTGGCTAGCA * \\
\hline swiM3 & M & intermediate & GCCTGAGTCCATGAGGGAAGAATATCAACAGGAACAGCAG * \\
\hline swiM4 & M & intermediate & AATGGGAGTGCAGATGCAGCGATTCAAGTGATCCTCTCGT * \\
\hline swiH1 & HA & intermediate & AAGACAAGCATAACGGGAAACTATGCAAACTAAGAGGGGT * \\
\hline swiH2 & HA & intermediate & GTGGAAACACCTAGTTCAGACAATGGAACGTGTTACCCAG * \\
\hline swiH3 & $\mathrm{HA}$ & intermediate & AAACGGCTGCTTTGAATTTTACCACAAATGCGATAACACG * \\
\hline swiN1 & NA & intermediate & CTTGGGAATCAAAATCAGATTGAAACATGCAATCAAAGCG * \\
\hline swiN2 & NA & intermediate & GTAAAGACAACAGTGTAAGAATCGGTTCCAAGGGGGATGT* \\
\hline swiN3 & NA & intermediate & CAGTCGCTTGGTCAGCAAGTGCTTGTCATGATGGCATCAA * \\
\hline swiN4 & NA & intermediate & TACGGCAATGGTGTTTGGATAGGGAGAACTAAAAGCATTA * \\
\hline $\mathrm{ABI} 04 \mathrm{mF}$ & M & PCR & TAACCGAGGTCGAAACGTA \\
\hline $\mathrm{ABI} 04 \mathrm{mR}$ & M & PCR & TTACTCTAGCTCTATGTTGACAAA \\
\hline $\mathrm{ABI} 05 \mathrm{hF}$ & HA & PCR & ATGAAGGCAATACTAGTAGTTCTGCT \\
\hline ABI05hR & HA & PCR & TTCTACACTGTAGAGACCCATTAG \\
\hline $\mathrm{ABI} 06 \mathrm{nF}$ & NA & PCR & ATGAATCCAAACCAAAAGATAATAA \\
\hline ABI06nR & NA & PCR & TTACTTGTCAATGGTAAATGGCAACT \\
\hline
\end{tabular}

* 25-mer poly (A) tail added at 3' end of each intermediate oligonucleotide. 
We observed that the matrix genes of each virus subtype tested were detected in all nanomicroarrays (seasonal H1N1, seasonal H3N2 and H1N1/2009) with varying signal profiles, and that the H1N1/2009 viral RNA could hybridize to the M02 and M03 capture oligonucleotides in the seasonal $\mathrm{H} 1 \mathrm{~N} 1$ and $\mathrm{H} 3 \mathrm{~N} 2$ arrays to produce a visible signal, indicating that cross hybridization occurred as would be expected [12]. We did not test H1N1/2009 samples from other geographical regions as it was difficult to obtain specimens during the study. However since the multiple capture oligonucleotides were designed from highly conserved sequences among the $\mathrm{H} 1_{09}$ and $\mathrm{N} 1_{09}$ genes, we predict that the current H1N1/2009 array is highly likely to detect pandemic influenza H1N1/2009 not only from California but also from other geographic regions. In this proof of concept experiment, we demonstrate that the $\mathrm{H} 1 \mathrm{~N} 1 / 2009$ array is highly specific for the detection of swine-origin influenza A virus and can be used to differentiate between H1N1/2009 and H1N1, H3N2 and H5N1 viruses.

Considering that influenza viruses can evolve rapidly and are capable of adapting and generating diversity through cross-species transmission and genetic mutation, their ability to adapt raises the risk of the emergence of novel influenza viruses to which humans are susceptible [10,13,14]. The identification of subtypes that are endemic in zoonotic sources or novel influenza strains that have pandemic potential is of high concern. The development of new virus detection systems is always important in reducing the risk of transmitted infectious diseases. However, it is extremely challenging to design assays that cover varying virus populations and newly emerging viral subtypes for rapid and accurate detection. We developed a PCR-free nanomicroarray detection platform using a capture/intermediate oligonucleotide strategy, allowing the coverage of a wide range of influenza A virus subtypes including H1N1, H3N2, and H5N1. The multiple capture and intermediate oligonucleotides are designed in conserved regions of each of the three genes spanning the whole genome in each subtype to ensure the target genes can be captured and detected, despite variations among the different strains. This type of $40 \mathrm{bp}$ oligonucleotide is able to detect minor viral variants with high specificity. We employed multiplexed intermediate oligonucleotides for many of the influenza A viruses to capture and detect the specific influenza virus subtypes, mitigating the risk of unidentified mutations. Although the current nanomicroarray method is less sensitive than the Taqman and PCR assay, this assay appears to possess the potential to be used as a method for the rapid detection, subtyping, and screening of newly emerging influenza viruses in one simple assay. This assay has many advantages since it is not limited by the need for bioenzymatic-based gene amplification, sequencing, culturing, or HA/NA subtyping of emerging strains.

The genetic differences between the HA and NA gene segments of H1N1/2009 and other H1N1 strains $[10,15]$ was significant enough to allow us to generate the multiple specific capture and intermediate oligonucleotides so that the $\mathrm{H} 1 \mathrm{~N} 1 / 2009$ virus can easily and rapidly be distinguished from other H1N1, H3N2, and H5N1 viruses. Although the nanomicroarray images can be scanned for further statistical analysis, conclusions on virus identity can be drawn from results seen as a specific intensity pattern by the naked eye. Our results therefore suggest this nanoparticle-based genomic microarray may provide a practical tool for virus identification, and may be particularly useful during emerging influenza pandemics. 


\section{Experimental Section}

\subsection{Designing Oligonucleotides}

Multiple capture and intermediate oligonucleotides complementary to the conserved regions of the $\mathrm{M}, \mathrm{H} 1, \mathrm{H} 3, \mathrm{H} 5, \mathrm{~N} 1$, and $\mathrm{N} 2$ genes for the H1N1, H3N2, and H5N1 influenza A viruses were designed as previously described [12]. Since the $\mathrm{HA}\left(\mathrm{H}_{09}\right)$ and NA $\left(\mathrm{N} 1_{09}\right)$ genes of $\mathrm{H} 1 \mathrm{~N} 1 / 2009$ are $27.2 \%$ and $18.2 \%$ different in amino acid sequence, respectively, to the $2008 \mathrm{H} 1 \mathrm{~N} 1$ strain [15], we selected 133 and 139 known sequences of $\mathrm{H}_{09}$ and $\mathrm{N}_{09}$ gene, respectively, including sequences of viruses from different geographical regions (i.e., Northern American, Europe, and Asia) and applied 500 hit count analysis for each gene using Blast Search program in Vector NTI Advance ${ }^{\mathrm{TM}} 11$ (Invitrogen, Foster City, CA, USA). The sequence alignment using the MEGA 4 program indicated that the oligonucleotides sequences we designed were highly conserved among the $\mathrm{H} 1_{09}$ and $\mathrm{N} 1_{09}$ genes. The nucleotide sequences of $\mathrm{H} 1$ and $\mathrm{H}_{09}$ or $\mathrm{N} 1$ and $\mathrm{N}_{09}$ were further aligned to identify significant divergent regions. The specific capture and intermediate oligonucleotides unique in H1N1/2009 were designed to diagnose and distinguish the H1N1/2009 from seasonal H1N1 influenza A viruses. A total of 11 specific capture and positive control oligonucleotides were synthesized and printed on the microarray chip. Three or four intermediate oligonucleotides that bind to sequences adjacent to the capture sequence for each target gene were designed and synthesized. To validate the H1N1/2009 nanomicroarry assay, the PCR primers for the $\mathrm{H}_{109}, \mathrm{~N}_{109}$, and $\mathrm{M}_{09}$ genes of $\mathrm{H} 1 \mathrm{~N} 1 / 2009$ were also generated to amplify full-length DNA fragments. The oligonucleotides sequences are listed in Table 1.

\subsection{Samples, Viral RNA Extraction and RT-PCR}

Influenza virus isolates A/Singapore/63/04 (H1N1), A/Hong Kong/8/68(H3N2), A/Brisbane/10/2007(H3N2), A/Vietnam/1203/04 (H5N1), were prepared at CBER, FDA using appropriate safety protocols. Swine-origin influenza A viruses A/California/04/2009 (H1N1), A/California/07/2009 (H1N1), A/California/08/2009 (H1N1), and the plasmids pHW-PR8 M (H1N1), pCR-II Topo HA (H5N1) and pCR-II Topo NA (H5N1) for HA, NA and M genes were provided by CDC (CDC, Atlanta, GA, USA). Some influenza virus strains were purchased from ZeptoMetrix (ZeptoMetrix corp., Buffalo, NY, USA). Genomic viral RNA was extracted directly from allantoic fluid or cell culture supernatant with QIAamp Viral RNA Mini Kit (QIAGEN, Valencia, CA, USA). The purified RNA was quantified using NanoDrop (NanoDrop Technologies, Inc., Wilmington, DE, USA). The standard RT-PCR was performed as described previously to amplify the $\mathrm{H} 1_{09}, \mathrm{~N} 1_{09}$, and $\mathrm{M}_{09}$ fragments.

\subsection{Nanomicroarray Assay}

Nanomicroarray slides for seasonal H1N1, H3N2, and H1N1/2009 were printed separately, and each capture oligonucleotide was arrayed on CodeLink Activated slides in the triple-spot format (Figure 1A) using the OMNIGrid Accent printer (Genomic Solutions Inc., Marlboroug, MI, USA). Each slide contained 10 identical sub-arrays partitioned by a hybridization gasket, thus enabling 10 tests per slide. Aqueous DNA-modified gold NP-probe and silver staining solutions were purchased 
from Nanosphere Inc (Northbrook, IL, USA). RNA/DNA samples and $10 \mathrm{nM}$ of the intermediate oligonucleotides were mixed in $100 \mu \mathrm{L}$ of the hybridization buffer containing $5 \times \mathrm{SSC}, 0.05 \%$ sorbitan mono-9-octa decenoate poly(oxy-1,1-ethanediyl), 0.05\% Tween-20 and 40\% formamide, and applied to the nanomicroarray incubating for $90 \mathrm{~min}$ at $40{ }^{\circ} \mathrm{C}$ with shaking at $500 \mathrm{rpm}$. After three washes in wash buffer $\mathrm{A}\left(0.5 \mathrm{~N} \mathrm{NaNO}_{3}, 0.01 \%\right.$ SDS and $0.05 \%$ Tween-20) and rinsed once in wash buffer $\mathrm{B}$ $\left(0.5 \mathrm{~N} \mathrm{NaNO}_{3}\right)$, the universal NP-probe was incubated with the slide for $30 \mathrm{~min}$ at $40{ }^{\circ} \mathrm{C}$. Then, the slides were stained with the Silver Enhancer A and B solutions for $5 \mathrm{~min}$. The light-scattering signal produced by silver-enhanced gold NPs was captured by a photosensor and converted to a TIFF image by a Verigene Reader [16]. The resulting TIFF images were analyzed using GenePix Pro 7 software [12,17].

\section{Conclusions}

In summary, this study provides evidence that the nanomicroarray assay may be able to differentiate genetic variations among influenza viruses when appropriate samples are available. The assay was able to detect H1N1/2009 and distinguish it from other influenza A viruses. This new method may be useful for simultaneous detection and subtyping of circulating human influenza A viruses and can be rapidly modified to detect other emerging influenza strains in public health settings.

\section{Acknowledgments}

The authors wish to acknowledge Krishnakumar Devadas, Mingjie Zhang and Robin Biswas for review of the manuscript. This work was a collaboration with Nanosphere, Inc. through CRADA (\#147-09), and was funded through CBER intramural and FDA MCM funds. The findings and conclusions in this article have not been formally disseminated by the Food and Drug Administration and should not be construed to represent any Agency determination or policy

\section{Conflict of Interest}

The authors declare no conflict of interest.

\section{References and Notes}

1. Webster, R.G.; Bean, W.J.; Gorman, O.T.; Chambers, T.M.; Kawaoka, Y. Evolution and ecology of influenza A viruses. Microbiol. Rev. 1992, 56, 152-179.

2. Rohm, C.; Zhou, N.; Suss, J.; Mackenzie, J.; Webster, R.G. Characterization of a novel influenza hemagglutinin, H15: Criteria for determination of influenza A subtypes. Virology 1996, 217, 508-516.

3. Fouchier, R.A.; Munster, V.; Wallensten, A.; Bestebroer, T.M.; Herfst, S.; Smith, D.; Rimmelzwaan, G.F.; Olsen, B.; Osterhaus, A.D. Characterization of a novel influenza A virus hemagglutinin subtype (H16) obtained from black-headed gulls. J. Virol. 2005, 79, 2814-2822.

4. Hilleman, M.R. Realities and enigmas of human viral influenza: pathogenesis, epidemiology and control. Vaccine 2002, 20, 3068-3087.

5. Nicholson, K.G.; Wood, J.M.; Zambon, M. Influenza. Lancet 2003, 362, 1733-1745. 
6. Gabriel, G.; Dauber, B.; Wolff, T.; Planz, O.; Klenk, H.D.; Stech, J. The viral polymerase mediates adaptation of an avian influenza virus to a mammalian host. Proc. Natl. Acad. Sci. U. S. A. 2005, 102, 18590-18595.

7. Hatta, M.; Gao, P.; Halfmann, P.; Kawaoka, Y. Molecular basis for high virulence of Hong Kong H5N1 influenza A viruses. Science 2001, 293, 1840-1842.

8. Tran, T.H.; Nguyen, T.L.; Nguyen, T.D.; Luong, T.S.; Pham, P.M.; Nguyen, V.C.; Pham, T.S.; Vo, C.D.; Le, T.Q.; Ngo, T.T.; et al. Avian influenza A (H5N1) in 10 patients in Vietnam. New Engl.J. Med. 2004, 350, 1179-1188.

9. Briand, S.; Mounts, A.; Chamberland, M. Challenges of global surveillance during an influenza pandemic. Publ. Health 2011, 125, 247-256.

10. Vijaykrishna, D.; Poon, L.L.; Zhu, H.C.; Ma, S.K.; Li, O.T.; Cheung, C.L.; Smith, G.J.; Peiris, J.S.; Guan, Y. Reassortment of pandemic H1N1/2009 influenza A virus in swine. Science 2010, $328,1529$.

11. Kitikoon, P.; Vincent, A.L.; Gauger, P.C.; Schlink, S.N.; Bayles, D.O.; Gramer, M.R.; Darnell, D.; Webby, R.J.; Lager, K.M.; Swenson, S.L.; et al. Pathogenicity and transmission in pigs of the novel $\mathrm{A}(\mathrm{H} 3 \mathrm{~N} 2) \mathrm{v}$ influenza virus isolated from humans and characterization of swine H3N2 viruses isolated in 2010-2011. J. Virol. 2012, 86, 6804-6814.

12. Zhao, J.; Tang, S.; Storhoff, J.; Marla, S.; Bao, Y.P.; Wang, X.; Wong, E.Y.; Ragupathy, V.; Ye, Z.; Hewlett, I.K. Multiplexed, rapid detection of H5N1 using a PCR-free nanoparticle-based genomic microarray assay. BMC Biotechnol. 2010, 10, 74.

13. Kawaoka, Y.; Krauss, S.; Webster, R.G. Avian-to-human transmission of the PB1 gene of influenza A viruses in the 1957 and 1968 pandemics. J. Virol. 1989, 63, 4603-4608.

14. Lindstrom, S.E.; Cox, N.J.; Klimov, A. Genetic analysis of human H2N2 and early H3N2 influenza viruses, 1957-1972: Evidence for genetic divergence and multiple reassortment events. Virology 2004, 328, 101-119.

15. Gallaher, W.R. Towards a sane and rational approach to management of Influenza H1N1 2009. Virol. J. 2009, 6, 51.

16. Tang, S.; Zhao, J.; Storhoff, J.J.; Norris, P.J.; Little, R.F.; Yarchoan, R.; Stramer, S.L.; Patno, T.; Domanus, M.; Dhar, A.; et al. Nanoparticle-Based biobarcode amplification assay (BCA) for sensitive and early detection of human immunodeficiency type 1 capsid (p24) antigen. J. Acquir. Immune Defic. Syndr. 2007, 46, 231-237.

17. Axon GenePix, Pro 7; MDS Analytical Technologies Inc.: Sunnyvale, CA, USA, 2008.

(C) 2012 by the authors; licensee MDPI, Basel, Switzerland. This article is an open access article distributed under the terms and conditions of the Creative Commons Attribution license (http://creativecommons.org/licenses/by/3.0/). 